\title{
Corrigendum
}

\section{CXCL11-dependent induction of FOXP3-negative regulatory T cells suppresses autoimmune encephalomyelitis}

Yaniv Zohar, Gizi Wildbaum, Rostislav Novak, Andrew L. Salzman, Marcus Thelen, Ronen Alon, Yiftah Barsheshet, Christopher L. Karp, and Nathan Karin

Original citation: J Clin Invest. 2014;124(5):2009-2022. https://doi.org/10.1172/JCI71951.

Citation for this corrigendum: J Clin Invest. 2018;128(3):1200-1201. https://doi.org/10.1172/JCI120358.

The Editors recently posted an Expression of Concern for this article due to duplication of some of the flow cytometry plots in Figures 5C and 7A (1). The authors have completed three replicate experiments for the panels in question, and the updated findings appear below. The revised experiments were conducted by Yaniv Zohar, and the analysis was completed independently in a blinded fashion.

Figure 5C
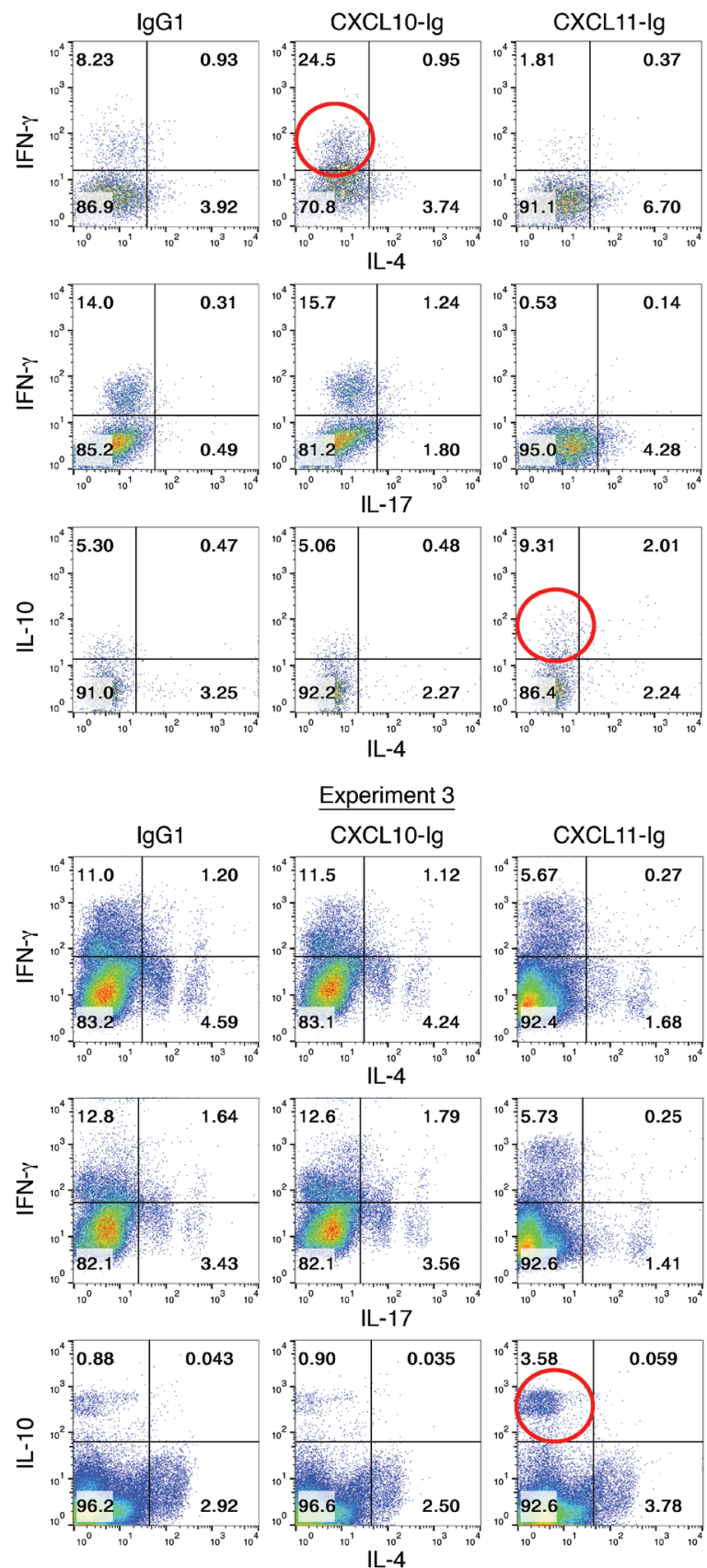

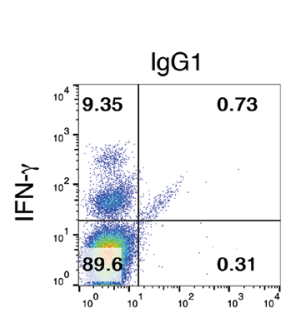

Experiment 2
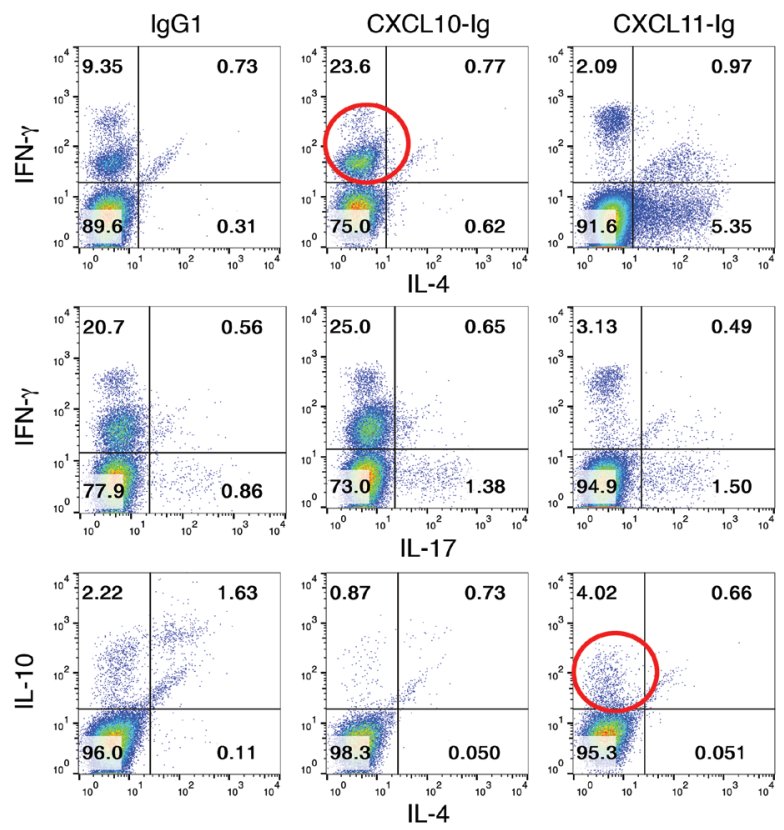

Summary of 3 samples from 3 experiments

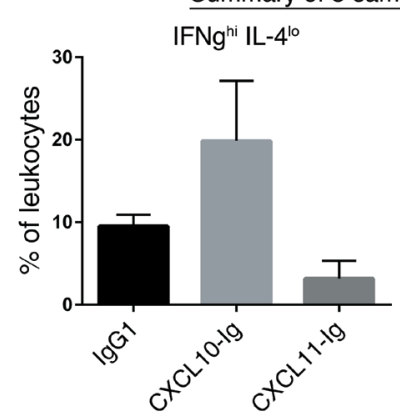

IFNg ${ }^{\text {lo }}$ IL-1 $17^{\text {hi }}$
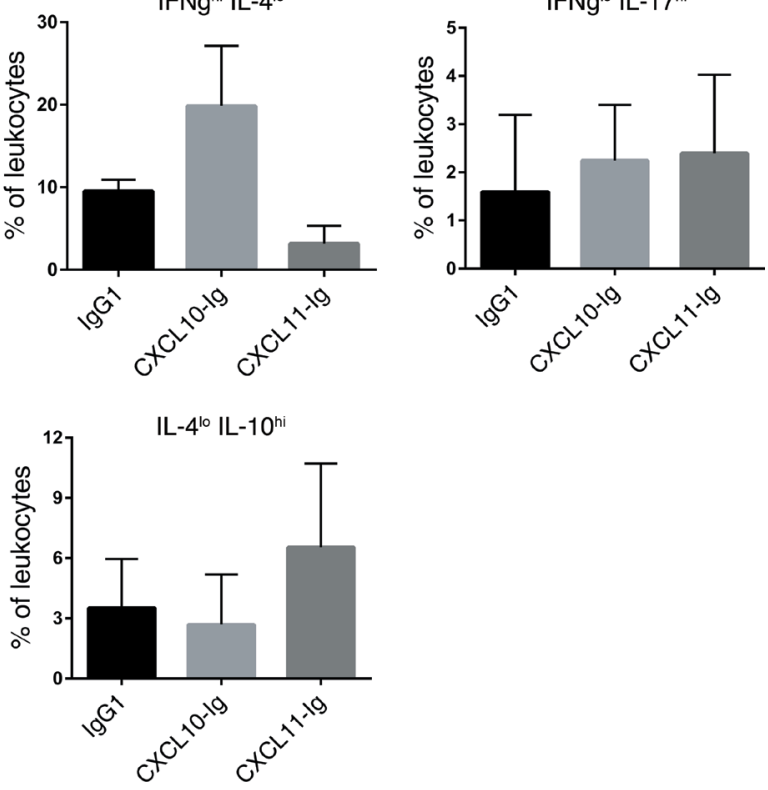
The authors report in Figure 5C that repeated administration of CXCL11-Ig increased the in vivo polarization of IL-10 ${ }^{\text {hi }}$ CD $4{ }^{+}$T cells, suggesting that CXCL11 supports Tr1 polarization. In addition, the authors state that Experiments 1 and 2 suggest that the administration of CXCL10-Ig induced IFN ${ }^{\text {hi }}$ Th1 cells, consistent with the notion of the differential functions of CXCL10 and CXCL11 in T cell polarization. This finding was not confirmed in Experiment 3.
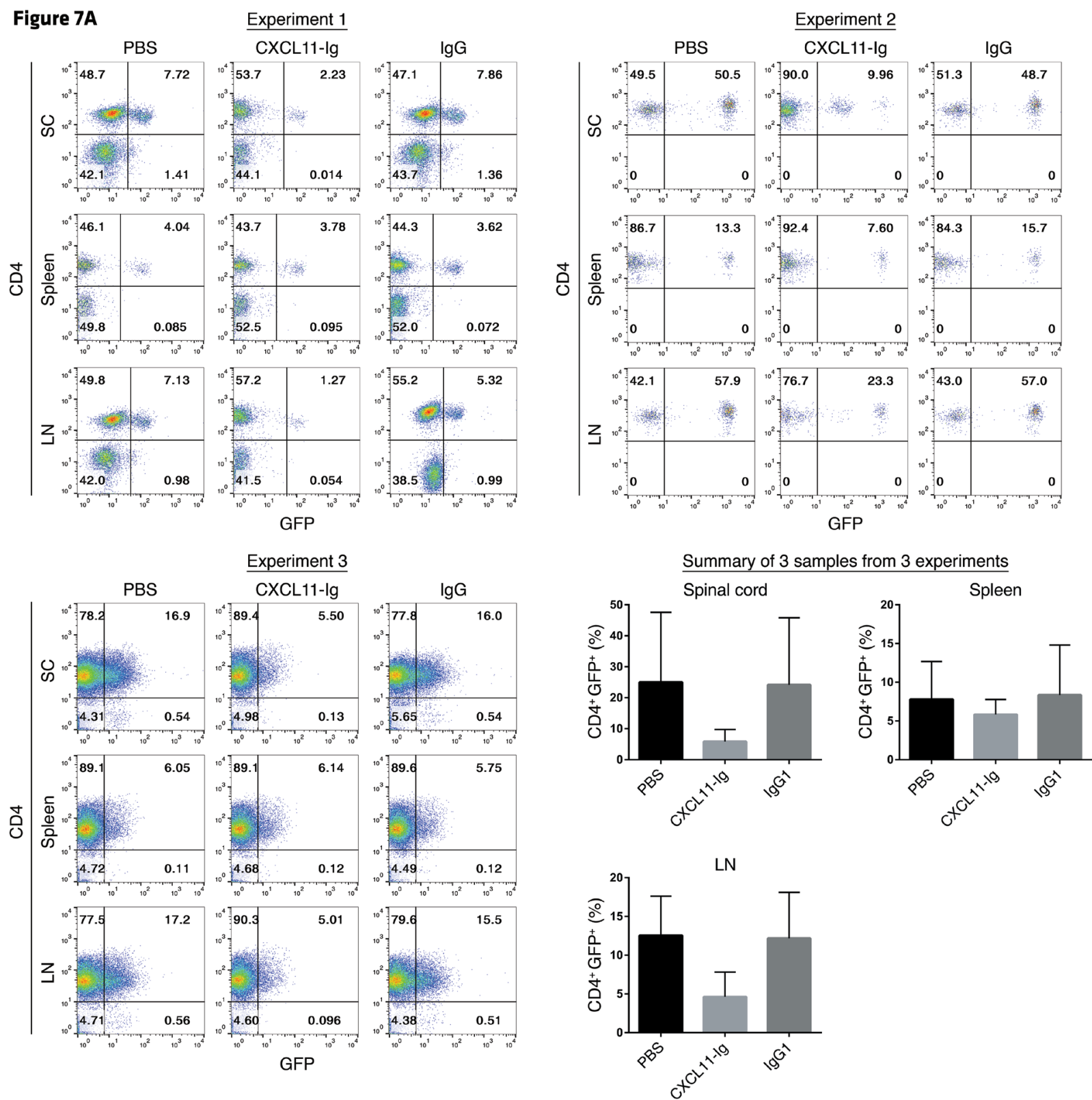

Figure 7A shows reduced accumulation of injected CD4 $4^{+} \mathrm{T}$ cells in the draining LN and spinal cord, but not the spleen, consistent with the original findings of the article.

The authors regret the errors and appreciate the opportunity to correct the article.

1. J Clin Invest. 2017;127(10):3913. https://doi.org/10.1172/JCI97015. 